\title{
Mental Disorders are Not Real: Using Skepticism and Critical Thinking to Challenge Key Myths in the Science of Mental Health
}

Pashak TJ*

Department of Psychology, Saginaw Valley State University, USA

\begin{abstract}
This review aims to identify and correct several prevalent misunderstandings about mental health and mental disorders which are potentially damaging to society. Organized into themes around four myths about mental disorders, this discussion provides readers with helpful information and references to other useful readings to support their skepticism and critical thinking about psychopathology. The four myths identified here are that mental disorders are unimportant, are solely biomedical entities, are only treatable with medication or CBT, and are real. In each case, the author makes a reasoned argument to the contrary, culminating in a discussion about how mental disorders are actually not real, but are nevertheless very important considerations. Throughout the discussion several logical fallacies are introduced (e.g., confusing correlation with causation, the nominal fallacy, the reification fallacy), as well as brief historical notes on the field of psychology (e.g., varying treatment modalities, schools of thought around treatment impactfulness, and problems with language progression in research).
\end{abstract}

Keywords: Mental disorders; Myth; Diagnosis; Psychotherapy; Effectiveness

\section{Mental Disorders are Not Real: Using Skepticism and Critical Thinking to Challenge Key Myths in the Science of Mental Health}

In this brief manuscript, I aim to identify several critical misunderstandings about mental health and provide readers with information to correct these common errors. My hope is that this review may serve as a useful reading resource for undergraduate students enrolled in courses such as Abnormal Psychology or Introduction to Clinical Psychology, as a prompt for critical thinking discussion for graduate students in clinical/counseling psychology or social work, as a quick teaching tool for psychiatry training, or even as a helpful reminder for those already engaged in the helping professions. By no means are the myths listed here a comprehensive or empirically-derived list of all significant problems in the science of psychopathology; however, they are a set of important and damaging assumptions I find myself addressing repeatedly with my own students and clients. Thus, I felt the need to compile these issues into an easily shared open-access format. Read on for a short list of key myths about mental disorders and ways to challenge those faulty beliefs.

\section{Myth \# 1: Mental Disorders are Unimportant}

This is absolutely false. While most people taking the time to read this article would probably not endorse this belief, there nevertheless exists a prevalent viewpoint which devalues the science of psychopathology and individuals' mental health experiences, and is dismissive of psychology in general. Themes commonly bundled into this belief include the notions of blame (e.g., "he's not depressed, he's just lazy"), disinterest (e.g., "why would I care about someone's touchy-feely issues?"), distrust (e.g., "I think those labels are just excuses"), prejudice (e.g., "I won't associate with crazies like her"), minimization (e.g., "it's a waste of time and energy to consider those things"), illegitimacy (e.g., "psychology isn't a real science like chemistry or physics"), disbelief (e.g., "they just act that way for attention"), antipathy/apathy (e.g., "they need to pull it together, they've got it no harder than everyone else") and possibly even fear (e.g., "that neighbor seems disturbed, maybe he's dangerous"). For many laypeople, these and other similar themes drive a general sense of unimportance regarding mental health and mental disorder.

Why do many outside the field disregard mental disorders despite their conceptual value as meaningful and important tools for understanding others? Numerous explanations can contribute, including Lilienfeld's themes of seeing psychology as common sense, perceiving a lack of scientific rigor, denying generalizations due to individual uniqueness, claiming the results are non-replicable, seeing findings as imprecise, and being unable to see the utility of the field [1]. Specific to the public devaluing of mental disorders as helpful constructs, I would propose two additional explanations: anecdotal thinking and difficulties with empathy. Regarding the former, I would argue that many who experience mental health throughout much of life simply have no lived experience from which to understand the mental unhealth of others, and therefore determine that something about the idea of disorders must be dishonest or otherwise amiss. In other words, they engage in the hasty generalization fallacy (e.g., "Since I've never had those thoughts/behaviors/experiences, I don't see why anyone else would), extrapolating from their lived experience of $n=1$ to build a general rule by which they perceive all others in the world. Regarding the latter, I would argue that empathy (for those mentally unhealthy) is simply difficult for many to muster. Without first-hand experience, relevant observations of close friends or family members, or extensive training, people likely find great challenge in trying to understand the thoughts/feelings/behaviors of those struggling with mental health problems. Indeed, research has shown that deep and accurate empathy on other topics (e.g., experiences of racism) is hard work and only

*Corresponding author: Travis J Pashak, Ph.D, Assistant Professor of Psychology, Saginaw Valley State University, USA, Tel: 989-964-2696; E-mail: tjpashak@svsu.edu

Received July 02, 2017; Accepted July 26, 2017; Published August 02, 2017

Citation: Pashak TJ (2017) Mental Disorders are Not Real: Using Skepticism and Critical Thinking to Challenge Key Myths in the Science of Mental Health. J Ment Disord Treat 3: 140. doi:10.4172/2471-271X.1000140

Copyright: (c) 2017 Pashak TJ. This is an open-access article distributed unde the terms of the Creative Commons Attribution License, which permits unrestricted use, distribution, and reproduction in any medium, provided the original author and source are credited. 
results from active perspective-taking and exposure [2].

While there may be numerous reasons for public denial of mental disorders, the evidence is clear that mental health problems are in fact common, important considerations, and are related to a plethora of human difficulties. For instance, epidemiological data show that approximately one quarter of American adults experience a mental disorder in a given year, nearly half will experience a mental disorder some time in life, and that suicide is a leading cause of death worldwide $[3,4]$. Further, the World Health Organization estimates that mental disorders fall in the top 20 causes for disability, and are also a significant source of lost years of life, particularly for women and racial minorities [5]. Lastly, the worldwide cost of mental illness has been estimated at a staggering 2.5 trillion US\$ as of 2010 [6]. Certainly, mental health and mental disorders are important considerations in making sense of the human condition.

\section{Myth \#2: Mental Disorders are Biomedical Problems}

This is false; or at least mostly false. Although the psychiatric approach, the traditional "medical model" of diagnostics, television advertisements for prescription psychoactive medications, and many other sources might lead to the belief that disordered mental health is entirely a biomedical issue (i.e., of the body and rooted solely in biological/genetic factors), this is simply not the case. While the general public and certain training contexts may have a truncated perspective, the majority of contemporary clinicians and researchers in psychopathology value what is called the biopsychosocial model [7]. This model is a collective set of findings from biological approaches (e.g., biochemistry, genetics, nutrition, neurology), psychological approaches (e.g., cognition, behaviorism, development, personality, psychoanalysis), and social approaches (e.g., sociology, cultural anthropology, politics, history), which all contribute to the understanding of mental health and mental disorder. To view mental disorders as strictly somatic/ bodily constructs is to completely disregard two-thirds of this widely accepted model. Further, some researchers have actually found evidence that it is damaging to view mental disorders this way. Specifically, one study showed that those who held biogenetic explanations of mental disorders were more fearful of and pessimistic for those struggling with mental health problems, which could serve to exacerbate stigma [8].

Some readers may be frustrated at this point, having believed for years that depression, for instance, is caused by a "chemical imbalance." This and other similar attributions for mental disorders fail to utilize the entirety of the biopsychosocial model, and may engage in confusion of correlation versus causation. While it is true that, since the 1960's and 1970's, the monoamine theory of depression (e.g., serotonergic malfunctioning and norepinephrine depletion) gained significant attention as a possible explanation for mood problems [9], contemporary psychiatric research concludes that "simple biochemical theories that link low levels of serotonin with depressed mood are no longer tenable" [10]. This is in part because the logic is seriously flawed. Finding that a neurochemical pattern is related to a behavioral pattern (i.e., correlation) does not imply that the neurochemical pattern precedes, explains, and directly influences the behavioral pattern (i.e., causation). Rather, the evidence appears to show that, sometimes, those two features co-occur. Further, even in causal laboratory studies of induced serotonin depletion resulting in depressive symptoms, the findings do not show that all depressive episodes are caused by faulty neurotransmitter patterns - just that this phenomenon may be a part of the explanatory puzzle. Extremely compelling evidence for the etiology (i.e., cause) of depression comes from other approaches within the biopsychosocial model; consider for instance that approximately $66 \%$ of those meeting criteria for Major Depressive Disorder in the firstwave National Comorbidity Survey were dealing with bereavement or some other recent major loss [11]. If someone experiences the death of their child, divorce from their spouse, or loss of their job, then it seems quite illogical to blame their subsequent depressive mood on faulty serotonergic functioning.

Major Depressive Disorder is hardly the only mental disorder to defect from the solely biomedical conceptualization. For instance, Schizophrenia, largely considered the "poster child" of psychiatric biomedically-oriented mental health problems, is still only approximately $40-50 \%$ genetically heritable, leaving much of the etiology to other psychosocial influences or other non-genetic factors (e.g., advanced paternal age, urban birth, migrant status, and prenatal infection) [12]. None of this is to say, of course, that biomedical variables are irrelevant- just that they are not the only factors involved. In other words, psychology (and therefore psychopathology) must be approached with an appreciation for both its natural science and social science components [13]. As an example, consider the widely utilized diathesis-stress model [14], which combines nature and nurture in its attempt to explain the causes of mental disorders. Originally formulated to address the etiology of Schizophrenia, but later applied to most other disorders, the diathesis-stress model postulates that a combination of diatheses and stressors can be used to statistically predict the likelihood of a mental disorder occurring. In this model, diathesis refers to one's personal vulnerabilities, disposition/personality/character traits, early formative experiences, and genetically inherited probability of a disorder. Thus, one's diathesis is their cumulative risk level for a certain disorder, given early/lifelong factors. Then, stress refers to current or recent difficulties, negative life events, challenging situations, injury/ change to the central nervous system, emotional strain, and even trauma. Thus, one's stress is the cumulative level of difficulty currently being faced. So together, the diathesis-stress model argues that diatheses (such as genetic vulnerability) are not the sole explanation for mental disorders, rather, they interact with individuals' current stress levels to influence the possibility of distress and impaired functioning. Some use the analogy of a "loaded gun" to help explain this model, such that some individuals are more "loaded" for a disorder than others (i.e., they possess a stronger level of diathesis) but nevertheless the environment is necessary to "trigger" the disorder onset (i.e., push the stress level high enough for disordered problems to manifest). In any case, this empirically supported approach to etiology certainly relies on both biomedical and psychosocial influences.

Additionally, there is in fact an inherent indication in the term "mental disorders" which differentiates them from solely biomedical entities. That differentiator is the term disorder as opposed to the term disease [15]. This subtle distinction may be lost on many outside the field, but it is an important acknowledgment of the nature of the problems themselves. The term disease is used to refer to problems in human functioning which have a known/identifiable pathophysiological process - thus diseases have an objectively observable set of bodily indicators (e.g., breast cancer involves observable malignant cell growth in breast tissue; pneumonia involves observable lung tissue inflammation and accompanying consolidation of fluid in air sacs). A disorder, on the other hand, refers to a problem in human functioning which lacks a clear pathophysiological process - in other words, the working of that problem is either not grounded exclusively in observable bodily processes, or, current research is in conflict as to why the problem occurs. With this comparison in mind, the fact that most mental health problems are named disorders (e.g., Major Depressive 
Disorder, Generalized Anxiety Disorder, Posttraumatic Stress Disorder, Borderline Personality Disorder) or are given other similarly-defined terms such as syndrome (e.g., Munchausen Syndrome by Proxy (i.e., Factitious Disorder - Imposed on Another) and Restless Legs Syndrome), dysphoria/dysmorphia (e.g., Gender Dysphoria and Muscle Dysmorphia), and nervosa (e.g., Anorexia Nervosa and Bulimia Nervosa), seems to support the notion that these constructs are indeed not solely biomedical, as they lack any clear pathophysiologic process [16]. Mental disorders are certainly not to be solely understood as biomedical entities, but rather, are best explained utilizing all levels of the biopsychosocial model.

\section{Myth \#3: Mental Disorders Must be Treated with Medication or CBT}

Again, this is a commonly held notion which is mostly false. Psychoactive medications and/or cognitive behavioral therapy (CBT) can both be appropriate forms of treatment for certain mental health problems, but the issue here is that many assume those are the only legitimate options, and this is simply incorrect.

If the logic is sound against framing mental disorders as solely biomedical entities and instead utilizing the biopsychosocial model, as argued in the prior section, then one would be wise to approach treatment with similar thinking. Unfortunately, many in the general public and within the field have a woefully narrow view of which mental health treatments are appropriate. The overarching treatment message many receive from television/radio media, online advertisements, and other contemporary information sources (often including their primary care physicians) consists of a glowing recommendation for psychotropic substances such as Paxil (Paroxetine), Xanax (Alprazolam), Prozac (Fluoxetine), or Celexa (Citalopram) - with no mention of counseling, psychotherapy, support groups, or other routes to improvement. This misinformation is a serious detriment to society, as psychological treatments have much to offer.

Evidence abounds that psychotherapy is effective in the treatment of mental health problems. Since the first major meta-analysis in 1980 and onward, researchers have consistently found positive data supporting the notion that psychological treatments have a significant impact [17]. Recent meta-analytic studies have been similarly optimistic for psychological interventions. For instance, in the treatment of depression, average effect sizes for psychotherapy appear to fall around 0.73 [18], while effect sizes for antidepressant medications appear to fall around 0.31 [19], indicating that while both approaches can be impactful, there may be reason to prefer psychological treatments. Further, another recent meta-analysis of numerous studies occurring since the 1990's shows that psychological treatments are similar, and at times more effective (either immediately or a later follow-up) than psychoactive medications in the treatment of issues including stress, depression, panic attacks, and insomnia [20]. Importantly, my aim here is not to argue against the responsible use of psychotropic medication, when necessary (and many clinicians would agree that pharmacological treatment is needed for more severe/disabling forms of mental disorders, such as those in the psychotic or bipolar categories), but rather to showcase evidence that psychological treatments such as individual psychotherapy do deserve serious consideration as effective forms of intervention.

Within those willing to accept the evidence that psychological treatments are effective, many appear to hold attitudes falling into the second part of this myth: that only cognitive-behavioral therapy (CBT) is an appropriate form of treatment modality. This too is patently false. Territory wars over the statuses of effective or efficacious have raged in the field of psychotherapy for decades, with various models of treatment each experiencing their heyday. Psychoanalysis, psychodynamic psychotherapy, humanistic psychotherapy, interpersonal psychotherapy, existential psychotherapy, behavioral treatments and behavior modification, cognitive psychotherapy, CBT, feminist therapy, multicultural counseling, eclectic or integrative approaches, and a whole host of more recent additions to the counseling tradition (e.g., mindfulness-based psychotherapy, dialectical-behavior therapy, etc.) have all clamored for an ever-elusive standing as the unanimously agreed-upon "best practice." By all accounts, CBT is currently experiencing a period of popularity in the field's history, and with changes being made to the structures of health insurance and treatment provision in many countries, now is an important time to disseminate accurate information about these therapies.

Probably most importantly, I will point out that longstanding research has shown most/all psychotherapies to be effective, and to be not significantly different from one another in their level of effect (sometimes dubbed the "dodo bird verdict") [21, 22]. Although the theorists behind each modality of psychotherapy/counseling have extensively researched and prolifically written about all the nuances of their respective treatment's "bells and whistles," the practice is nevertheless primarily comprised of a set of common factors (i.e., shared among nearly all modalities) which appear to statistically account for a greater degree of variance than the unique factors. In other words, sitting down to talk with a professional who patiently listens, deeply cares, accurately empathizes, non-judgmentally explores, authentically validates, and gently challenges a client with mental health concerns (when, of course, the clinician is adequately trained in ethics, cultural competence, etc.) - this is meaningful and effective. Whether the clinician tends to focus most on thoughts, behaviors, relationships, interactions, memories, impulses, desires, fears, etc., these details may steer the content the of treatment but many not greatly alter the ultimate level of impact.

Despite the fact that many practicing clinicians are intimately aware of the dodo bird verdict and treat their clients accordingly, utilizing skills from the modalities best suiting their personalities and their clients' needs, there continues to exist a widespread push toward empirical "proof" of superiority for one model or another. Primarily led by academic researchers (who typically conduct psychological treatments far less frequently, if at all, compared to their peers in applied settings), this search for a gold standard has led to some rifts in the fields of psychopathology and clinical/counseling psychology. Of note is the contention around evidence of impact, and the forms of evidence which are acceptable. One "camp" approves of the EmpiricallySupported Treatments (EST) model, whereas the other camp approves of the Evidence-Based Practices (EBP) model. There is much literature on this divide [23, 24], but briefly, the EST model tends to focus on the evaluation of a treatment's efficacy (i.e., ability to alleviate a specific symptom or symptom cluster) through tightly controlled internally valid laboratory studies such as randomized clinical trials, whereas the EBP model tends to focus on the evaluation of a treatment's effectiveness (i.e., overall impact upon general improvement) through studies which more closely mirror real-life clinical work with external validity. Broadly, the EST model tends to value empirical data such as experiments and meta-analyses, whereas the EBP model would value that data plus other sources of clinical knowledge (e.g., clinical expertise, case studies, supervision, correlational data, etc.). So, the EST model tends to be narrower in how it delineates which treatments are valid. When CBT's heyday is combined with the current trend of hiring primarily 
CBT-oriented researchers to tenure-track positions in psychology, as well as other historical factors in the field, the consequence is that CBT treatments tend to receive privilege and priority over other modalities in terms of trainee exposure, frequency of publications, and available evidence.

At the crux of the EST/EBP rift is a debate over how to view clinical work, and how to view psychology in general. Academic researchers in psychology and exclusively-CBT practitioners (particularly those adhering to manualized therapies) typically orient themselves around a positivistic view of the world, where only tightly internally valid data can contribute to the discussion - all else would detract from psychology's stance as a science. This status preservation, keeping psychology aligned with the STEM fields, is so precious to some that it may significantly bias their views toward the field and its constituents' activities. For instance, even in a recent article in The Clinical Psychologist with a balanced-sounding title of "Epistemic Humility," the tone still centered around positivistic empiricism-only attitudes and the near exclusion (or even disregard) of humanistic/hermeneutic clinical work [25]. Many clinicians, however, appreciate and understand the scientific underpinnings of the field while also valuing the inherent art involved in the counseling relationship - a subjective, phenomenological, and experiential endeavor which may lose generalizability if placed under a microscope. With this in mind, it is no surprise that proponents of CBT (or generally EST) are likelier to engage in scientific research, publish articles, and promote the idea that CBT is superior- because proponents of psychodynamic, existential, or feminist therapies for example (and generally EBP) may be likelier to spend their time in the provision of psychological services, supervision of trainees, or writing theory-building articles rather than contributing to the growing EST literature. In other words, a lack of empirical evidence supporting a certain modality does not necessarily equate to support against that modality. And researchers outside the CBT tradition are indeed beginning to make an empirical impact. For instance, consider that a recent series of studies and meta-analyses on psychodynamic psychotherapy are highlighting evidence that the treatment modality is frequently equally impactful to CBT, more impactful than medications, and often yields long-term continued growth of functioning/insight at follow-up which other treatments may not offer, across a variety of types of concerns/disorders [26, 27, 28]. Certainly, non-CBT modalities have much to offer, as do non-individual modalities (e.g., couples' psychotherapy/counseling, family interventions, and group therapy). These alternatives to the now-popular individual-CBT approach should all be taken seriously as legitimate options for treating mental disorders.

Finally, I would also like to point out that a plethora of meaningful "treatments" exist outside of the realm of formalized psychiatric and psychological care, which can possibly contribute to mental health. For instance, many individuals can find growth through: improvement of their physical fitness (e.g., exercise classes, running, massage therapy, chiropractic, diet/nutrition management); exploration of their religiosity/spirituality (e.g., worship groups, scripture readings, yoga, pastoral counseling, prayer); enhanced social connection (e.g., activity clubs, anonymous substance-use meetings, grief support groups, online forums); and self-help (e.g., books, online materials, audio recordings). Mental disorders need not be treated exclusively with psychotropic medications or cognitive-behavioral therapy; rather, many other forms of psychological treatment are valid and effective.

\section{Myth \#4: Mental Disorders are Real}

Mental disorders are not, in fact, real. At least not in the concrete sense of the word "real." Before any readers become infuriated with me, remember that in the prior sections I argued that mental disorders are indeed important and that they can also be treated, so I am certainly not aiming to invalidate the mental health struggles of millions of people. Rather, the mythical belief I am addressing here is that many think of mental disorders as real things, pseudo-physical entities which are unique, classifiable, and can cause symptoms, and which therefore need to be cured. This line of thinking, while possibly comforting, is based largely on logical errors and fallacies.

\section{Collections, Not Causes}

First, consider how disorders are defined and described in the professional literature. The Diagnostic and Statistical Manual of Mental Disorders (DSM, currently in its fifth edition [16] gives a general definition focused on disturbance, clinical significance, and problems in thought, feeling, and/or behavior. Then, throughout the DSM, individual lists are provided which detail the symptoms of each of several hundred disorders. The leap in thought taken by many is that the disorders included in the DSM actually cause their respective symptom lists (as would be true for diseases which cause symptoms), but this is actually not the case. Rather, the list of symptoms is the disorder causality in any direction is not presumed. A disorder is really a collection of problems which often seem to co-occur; the etiology of this cluster of symptoms is a much larger discussion (which, as addressed earlier, calls upon the biopsychosocial model and diathesis-stress model rather than solely biomedical findings). This misunderstanding is prevalent throughout the general public and often within the field as well. Hearing someone say "my social anxiety makes it hard for me to speak in large groups" likely doesn't sound shockingly illogical at face value - but the thought process does in fact engage in a form of circular logic called the nominal fallacy (i.e., the belief that because something has been named, it has also been explained). The key word in that example is "makes," which implies that the individual's Social Anxiety Disorder (a named collection of symptoms involving discomfort over fear of judgment) somehow caused a specific experience to occur (feeling anxious with large crowds). As astutely commented by Jonathan Shedler, this logic "...makes no more sense than saying 'anxiety is caused by anxiety.' The label is not the cause." [29].

\section{Labels, Not Labeled}

Even if they are collections of symptoms, why can't disorders be causes? A crucial reason is that they are socially-constructed labels, rather than tangibly existent entities which come to be labeled. Pardon the cliché, but consider Shakespeare's commentary on a rose: by any other name (i.e., label), it would still remain a rose, and thus smell just as sweet. The rose itself is a real thing which exists in nature, in a tangibly observable way, which would continue to exist unchanged even if described/named/labeled differently by society. Mental disorders, on the other hand, do not exist in the natural world in some tangibly observable way (because as discussed earlier, they are not solely biomedical entities like tumors; they are complex biopsychosocial constructs). So, if a new DSM were written (as happens every several years) and a current disorder were edited to include different symptoms, then the entire construct would be changed and would no longer remain in its prior form (e.g., consider some of the changes made to the definition of "trauma" within Posttraumatic Stress Disorder, or the fact that Asperger's Syndrome was completely removed in DSM-5) [16].

\section{Categories, Not Classifications}

Part of the reason people have come to view mental disorders 
as real things, I believe, is due to the medicalized "hard-science" language developed to describe them, which is somewhat misleading. In the natural sciences, many phenomena can be classified into an organizational structure via unique characteristics (e.g., the classification of animals into their kingdom, phylum, class, order, genus, and species; the classification of physical materials by their composite parts using the periodic table of the elements). This clean and tidy arrangement of information is an appealing epistemology to many in the social sciences as well, and has often been attempted - the taxonomy of disorders in the DSM being a prime example. However, the organization of disorders differs from the organizing principles in the natural sciences in a very important way: the DSM is based on clusters of symptoms via self-report from those experiencing the symptoms (i.e., it is phenomenological) rather than being based on objectively observable characteristics as a true nosology would. Thus, in having to rely on subjective factors rather than unique natural traits, the disorder taxonomy is inherently less stable, prone to change over time, likely to have cluster-overlap, is hedged in majority culture rather than representing all humanity, and can only provide estimations of categories rather than legitimate classifications. This is why many students in undergraduate Abnormal Psychology classes are stunned when they hear that two individuals both diagnosed with Major Depressive Disorder may not technically share a single symptom; that the difference between Bipolar I and Bipolar II rests on a loose clinical judgement of "severity" of the already severe manic episode; that Posttraumatic Stress Disorder and Acute Stress Disorder basically are only separated by an arbitrary cutoff of 1 month; and that those with at least one mental disorder are likelier to have two or more rather than being uniquely classified with solely one label (i.e., the problem of comorbidity) [16].

\section{Constructs, Not Concrete}

A final form of misunderstanding, with regard to mental disorders being "real," is that some people begin with a reasonable grasp on disorders as constructs, but inadvertently slip into viewing them as concrete as time passes and discussion continues. To be clear, a construct is a hypothetical explanation used to aid in understanding when no existent observable process is available. Mental disorders are indeed constructs, and this is why they are referred to as "socially-constructed" (i.e., society, via the DSM and other sources, has constructed these labels). Synonyms for construct in this sense could include concept, idea, and notion. Most of the time, when faced with the statement "disorders are not real, they are constructs" people can initially follow along. However, before long, many will subtly revert back to assigning disorders the properties which only existent concrete objects can have. As Eacker explains it, many people (even psychological researchers) have a bad habit of "mistaking as things entities which are not things." [30]. This happens in part through a grammatical shift in language, which is then believed to be accurate. One specific form of this problem is called the reification fallacy, or the logical error of treating a construct as though it were real in nature. For instance, consider introversion: early social science researchers found interest in studying those who were shy, less-outgoing, less-assertive, etc., and decided upon the name "introverted" as an adjective to describe them (e.g., "he is introverted"). Then, before long, people began to discuss the term as a noun instead of an adjective (i.e., "he has the trait of introversion"). Lastly, as a noun, people begin to apply existent and causal properties to the construct, which is ultimately illogical (e.g., "his introversion caused him to stay at home instead of going to the party"). This subtle shift of the reification fallacy has an insidious tendency to manifest with many social science phenomena, and the notions of mental disorders are probably one of the strongest examples with real-world consequences. This process leads people to believe that mental disorders are real, existent, tangible, physical entities which can be possessed by individuals and which have causal powers - but this is all false. Just like an individual cannot possess love, but they can be in love; and love does not cause them to feel a certain way, rather, it is the label for how they feel, so too with psychopathology constructs: he does not have depression, he is depressed; his anxiety is not causing his symptoms, it is his symptoms. And while these distinctions may appear to be pedantic grammatical minutia at first glance, they can indeed have negative consequences. As researchers and clinicians alike have already begun to notice $[15,31]$, the reification of mental disorders has done a major disservice to the public, misinformed millions of consumers of mental health treatments, tarnished the scientific credibility of the field, and stymied research progress in psychopathology for decades.

\section{Conclusion}

In this manuscript, I have attempted to compile and critique numerous major misunderstandings about mental disorders, synthesized under the headings of four commonly held myths: that mental disorders are unimportant, that mental disorders are biomedical entities, that mental disorders can only be treated with medication or CBT, and that mental disorders are real. Given that some of my statements may come across as controversial, please allow me to be clear in reiterating my intent with this piece: I do not aim to invalidate or minimize the mental health struggles experienced by anyone - your perspective matters, your pain is real, and as a clinician myself I hope to be as empathic as possible. What I do hope to address are the misunderstandings about mental health and the science of psychopathology floating about in society, which have the potential to be damaging and to worsen an already contentious field. Thus, I felt compelled to find a way to package this discussion briefly and accessibly to various audiences of readers.

Considering the available research literature and theoretical writings, extensive clinical supervision and consultation, and first-hand experiences with clients and students alike, I feel confident in making the following conclusions: Mental disorders are extremely important considerations, and mental health work is deserving of parity with other forms of human health and functioning. Mental disorders are best viewed through multi-systems thinking, for instance using the biopsychosocial model, and other platforms which value the enormous complexity of the human condition and its many layers of function and dysfunction. Mental disorders (and more importantly, the humans experiencing mental unhealth) can be successfully and effectively treated through a variety of means, including psychotherapy/counseling which spans a history of various schools of thought, each with their valuable kernels of wisdom and insight. Finally mental disorders are not real but they are socially-constructed descriptive labels to be understood with nuance and empathy.

\section{References}

1. Lilienfeld SO (2011) Public skepticism of psychology: Why many people perceive the study of human behaviour as unscientific psychologist. 67: 111 . 129

2. Gutierrez B, Kaatz A, Chu S, Ramirezm D, Samson-Samuel C, et al. (2014) "Fair play": A videogame designed to address implicit race bias through active perspective taking. Games for Health Journal 3: 371-378.

3. Kessler RC, Berglund P, Borges G, Nock M, Wang PS (2005) Trends in suicide ideation, plans, gestures, and attempts in the United States. Journal of the American Medical Association 293: 2487-2495.

4. Kessler RC, Chiu WT, Demler O, Walters EE (2005) Prevalence, severity, and 
Citation: Pashak TJ (2017) Mental Disorders are Not Real: Using Skepticism and Critical Thinking to Challenge Key Myths in the Science of Mental Health. J Ment Disord Treat 3: 140. doi:10.4172/2471-271X.1000140

Page 6 of 6

comorbidity of 12-month DSM-IV disorders in the National Comorbidity Survey Replication. Archives of General Psychiatry 62: 617-627.

5. World Health Organization. The Global Burden of Disease: 2004 Update. Geneva, Switzerland: WHO Press, 2008.

6. Bloom DE, Cafiero ET, Jané-Llopis E, Abrahams-Gessel S, Bloom LR, et al (2011) The global economic burden of non communicabe diseases. Geneva: World Economic Forum.

7. Engel GL (1977) The need for a new medical model: A challenge for biomedicine. Science 196: 129-136.

8. Haslam N, Kvaale EP (2015) Biogenetic explanations of mental disorder: The mixed-blessings model. Current Directions in Psychological Science. 24: 399-404.

9. Schildkraut JJ (1965) The catecholamine hypothesis of affective disorders: A review of supporting evidence. The American Journal of Psychiaritry 122: 509-522.

10. Cowen PJ, Browning M (2015) What has serotonin to do with depression? World Psychiatry 14: 158-160.

11. Wakefield JC, Schmitz MF, First MB, Horwitz AV (2007) Extending the bereavement exclusion for major depression to other losses. Archives of General Psychiatry 64-433-440.

12. Gejman PV, Sanders AR, Duan J (2010) The role of genetics in the etiology of schizophrenia. Psychiatric Clinics of North America 33: 35-66.

13. Schwartz SJ, Lilienfeld SO, Meca A, Sauvigné KC (2016) The role of neuroscience within psychology: A call for inclusiveness over exclusiveness. American Psychologist 71: 52-70.

14. Zubin J, Spring B (1977) Vulnerability—A new view of schizophrenia. Journal of Abnormal Psychology 86: 103-126.

15. Hyman SE (2010) The diagnosis of mental disorders: The problem of reification. Annual Review of Clinical Psychology 6: 155-179.

16. American Psychiatric Association (2013) Diagnostic and statistical manual of mental disorders: DSM-5. Washington, D.C: American Psychiatric Association.

17. Smith ML, Glass GV, Miller TI (1980) The benefits of psychotherapy. Baltimore, MD: Johns Hopkins University Press.

18. Robinson LA, Berman JS, Neimeyer RA (1990) Psychotherapy for the treatment of depression: A comprehensive review of controlled outcome research. Psychological Bulletin 108: 30-49.

19. Turner EH, Matthews AM, Linardatos E, Tell RA, Rosenthal R (2008) Selective publication of antidepressant trials and its influence on apparent efficacy. New England Journal of Medicine 358: 252-260.

20. BarlowDH (2004) Psychological treatments. The American psychologist 59 869-878.

21. Luborsky L, Singer, Luborsky L (1975) Comparative studies of psychotherapies Is it true that "everyone has won and all must have prizes"? Archives of General Psychiatry 32: 995-1008.

22. Seligman MEP (1995) The effectiveness of psychotherapy. American Psychologist 50: 965-974.

23. American Psychological Association Presidential Task Force on EvidenceBased Practice (2006) Evidence-based practice in psychology. American Psychologist 61: 271-285.

24. Chambless DL, Ollendick TH (2001) Empirically supported psychologica interventions: Controversies and Evidence. Annual Review of Psychology 52: 685-716.

25. Lilienfeld SO, Lynn SJ, O'Donohue WT, Latzman RD (2017) Epistemic humility: An overarching educational philosophy for clinical psychology programs. The Clinical Psychologist 70: 6-14.

26. Shedler J(2010) The Efficacy of Psychodynamic Psychotherapy. American Psychologist 65: 98-109.

27. Leichsenring F, Abbass A, Gottdiener W, Hilsenroth M, Keefe JR, et al. (2016) Psychodynamic therapy: a well-defined concept with increasing evidence. Evidence Based Mental Health.

28. Fonagy P (2015) The effectiveness of psychodynamic psychotherapy: An update. World Psychiatry.

29. Shedler J (2013) Doublethink diagnosis 2.0: A psychiatric diagnosis cannot be the "cause" of anything. Psychology Today.

30. Eacker JN (1975) The problem of reification. In Problems of Philosophy and Psychology 33-39.

31. Greenberg G (2013) The book of woe: The DSM and the unmaking of psychiatry. New York, NY: Blue Rider Press. 